\title{
Results of the Consensus Platforms: Positions and Recommendations Derived from Scientific Findings in a Joint Effort of Academics, Industry, and Authorities
}

\author{
Marcel Trachsel ${ }^{\star}$
}

\begin{abstract}
To convert scientific findings of the National Research Program (NRP) 'Endocrine Disruptors' into political and practical action the innovative tool of the consensus platforms has been created. The consensus platforms brought together representatives of the NRP, of the authorities and industry. They prepared generally accepted positions on the effects of endocrine disruptors and recommendations on actions to be taken to minimize these effects. This article shows the positions and recommendations of all three consensus platforms.
\end{abstract}

Keywords: Consensus platforms · Endocrine disruptors · Positions · Recommendations

\section{Introduction}

Chemicals with endocrine activity, also termed 'endocrine disruptors', can exert a variety of detrimental effects on humans, animals or entire ecosystems. The National Research Program 'Endocrine Disruptors' aimed to develop scientific strategies to assess the risks and hazards that arise when endocrine disruptors are processed through ecosystems to cause human and animal exposure. Consensus platforms enabled representatives of the NRP, of the authorities
*Correspondence: Dr. M. Trachsel, Implementation Officer National Research Program 'Endocrine Disruptors'

int/ext Communications AG

St. Johanns-Vorstadt 22

$\mathrm{CH}-4004$ Basel

Tel.: + 41612648805

Fax: + 41612648801

E-mail: marcel.trachsel@int-ext.com and of the producing and applying industries to prepare recommendations on how to minimize the negative impact of endocrine disruptors on the basis of the most recent scientific knowledge. The consensus platforms are an innovative tool applied in this program to convert achievements of scientific work into political and practical action by creating generally accepted recommendations for handling and future development of endocrine disruptors.

In the time span from June 2005 to December 2007 we have organized and realized three consensus platforms.

\section{Definition}

The consensus platform was defined to be a stepwise moderated issue-driven constructive non-public debate among scientists, industrial decision makers (producing and applying industry) and decision makers from the relevant authorities. The purpose was to create a best general agreement upon the impact of endocrine disruptors on humans, animals and ecosystems and upon a set of recommendations to politics, industry and authorities.

\section{Methodology}

The methodology of the consensus platform was developed for the National Research Program 'Endocrine Disruptors' based on the objectives defined by the Steering Committee. It is a series of meetings and individual preparatory reflection (Fig.).

\subsection{Preconditions}

To be successful a consensus platform requires certain conditions to be met:

- The issue to be treated must be defined as narrowly as possible.

- The members are willing to cooperate within the limits of the process, to accept divergent interests and viewpoints and work together to achieve agreement.

- The meetings are moderated professionally.

- All developments and interim results are treated as confidential until the consensus platform is completed. On termination of the work, only approved results are communicated.

\subsection{Step 1: Definition of Individual Positions}

On the basis of their knowledge the members define their individual positions, or that of the organization they represent, on the impact and action of endocrine disruptors.

\subsection{Step 2: Exchange and Compilation of Positions}

At the first joint meeting the members of the consensus platform put across their 


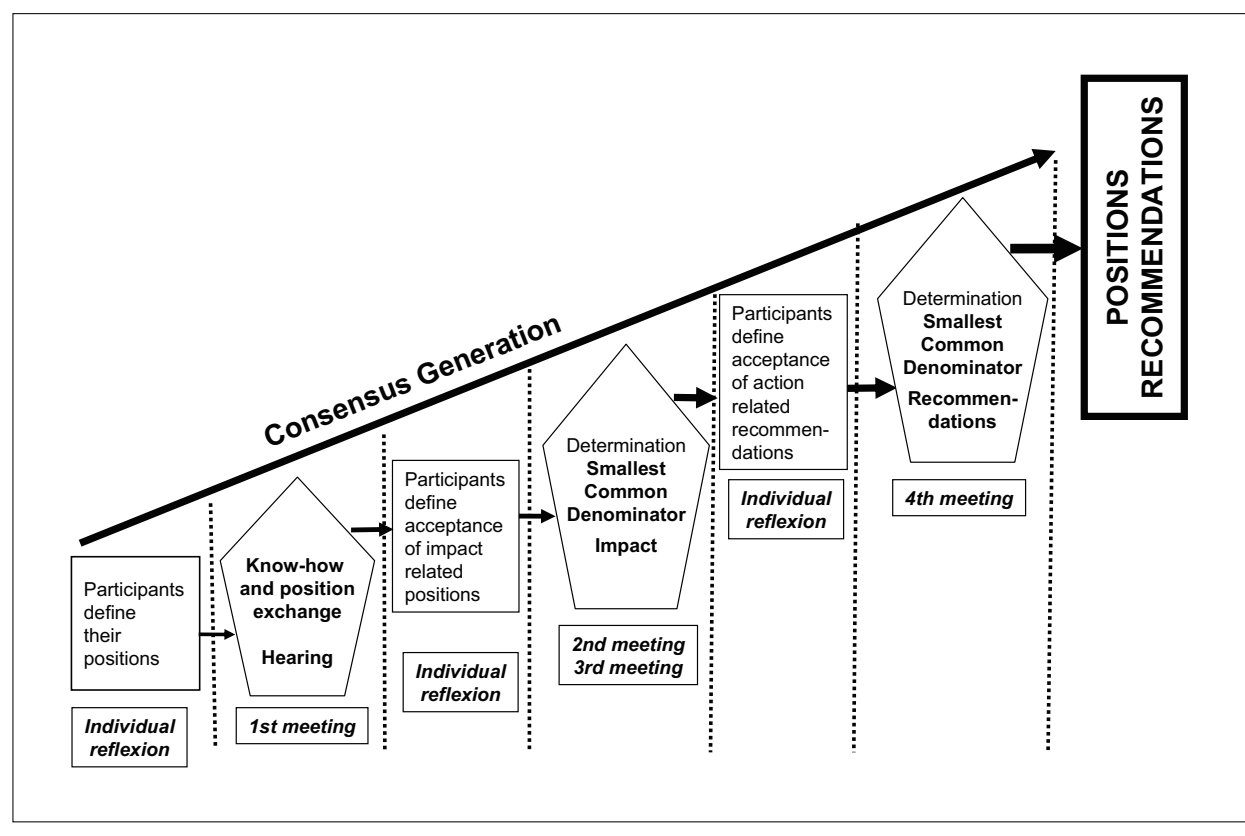

Fig. Methodology of Consensus Platforms

positions on the impact and action and respond to questions on their positions from other members of the consensus platform (hearing). On the basis of this meeting $a$ list of impact-related positions and one of action-related recommendations are compiled. These lists are then made available to the members of the consensus platform.

\subsection{Step 3: Individual Decision on Acceptance of Impact-related Positions}

In this step each member decides individually on acceptance, conditional acceptance or non-acceptance of each impactrelated position. In the event of conditional acceptance, conditions must be defined. The results are collected and categorized centrally as follows:

- Category i1: generally accepted $\gg>$ immediate inclusion in the final list of accepted positions

- Category i2: accepted or conditionally accepted, no more than one non-acceptance $>>$ to be discussed at the joint meeting

- Category i3: more than one non-acceptance $>>>$ is not further pursued

\subsection{Step 4: Meeting to Find General Agreement on Impact-related Positions}

At the joint meeting, the Category i2 impact-related positions are subjected to further discussion in order to find a formulation that is acceptable to all members of the consensus platform. The outcome of this meeting, together with the Category i1 positions, yields the final list of accepted impact related positions. These are set out in a logical sequence and approved by the consensus platform.

\subsection{Step 5: Individual Decision on Acceptance of Action-related Recommendations}

In this step each member of the consensus platform decides individually on the acceptance, conditional acceptance or non-acceptance of each action-related recommendation. In the event of conditional acceptance, condition must be defined. The results are collected and categorized centrally as follows:

- Category a1: generally accepted $\gg>$ immediate inclusion in the final list of accepted recommendations

- Category a2: accepted or conditionally accepted, no more than one non-acceptance $>>>$ to be discussed at the joint meeting

- Category a3: more than one non-acceptance $>>>$ is not further pursued

\subsection{Step 6: Meeting to Find General Agreement on Action-related Recommendations}

At the joint meeting, the Category a 2 action-related recommendations are subjected to further discussion in order to find a formulation that is acceptable to all members of the consensus platform. The outcome of this meeting, together with the Category a1 recommendations, yields the final list of accepted action-related recommendations. These are set out in a logical sequence and approved by the consensus platform.

\subsection{Step 7: Communication}

The approved lists of impact and action related statements are set out in a final document and made available for communication to other stakeholders.

\section{Application}

In the framework of the National Research Program 'Endocrine Disruptors' the methodology of the consensus platforms has been applied to three different issues, two substance-related and one mediumrelated: 'UV Filters in Sun Screens', 'Brominated Flame Retardants' and 'Endocrine Disruptors in Wastewater and in the Aquatic Environment'. With their joint effort to prepare impact- and action-related statements, representatives from producing and applying industry, regulatory authorities and scientists of the National Research Program 'Endocrine Disruptors' participated in a constructive dialogue, thereby making a valuable contribution towards preventing negative impacts of endocrine disruptors.

\section{Results of the Consensus Platform 'UV Filters in Sun Screens'}

The consensus platform 'UV Filters in Sun Screens' involved a total of 16 representatives of the producing and applying industries, the authorities and science. They accepted and approved 19 impact-related positions and 18 action-related recommendations.

\subsection{General Positions on the Endocrine Effect of Chemicals}

- There are chemicals that can influence the endocrine systems of organisms, including those of humans.

- There is a confirmed causal relationship between the occurrence of endocrine disruptors in ecosystems and certain detrimental effects in wild animals. It is postulated that biodiversity is impaired by endocrine disruptors. Endocrine disruptors such as tributyltin have led to the eradication of water snails from contaminated sites.

- Endocrine-like effects have been observed worldwide in fish exposed to effluent from biomechanical wastewater treatment plants. Endocrine disruptors have been clearly demonstrated in such water.

- Humans are exposed to endocrine disruptors in different ways; for many chemicals, little is known about their endocrine potential.

- Human beings are exposed to very low concentrations of endocrine disruptors. These chemicals may have a concerted effect. Many of them can be demonstrated in human tissue and in the maternal milk, so that human beings already come into contact with them in early stages of their life. Further scientific research is required in this area.

- Experimental in vitro and in vivo studies show that mixtures of different en- 
docrine disruptors exert an endocrine effect even if an effect cannot be demonstrated for the individual components in the given concentration.

- Steroid and thyroid hormones play an important part in biological effects and developmental processes. Contamination of the environment can have an effect on these systems. In the case of polychlorinated biphenyls (PCBs) this has been demonstrated in humans. A great deal of progress has been made in identifying new endocrine substances. The new substances include chemicals such as certain UV filters and certain antioxidants in cosmetics and preservatives in food. Further scientific research is required.

- The mechanisms of early sexual development are similar in a large number of mammals. Observations in animals can therefore yield pointers to effects on humans.

- The problems raised by endocrine disruptors necessitate long-term monitoring and research measures.

- There is a lack of validated study protocols.

- Scientific uncertainty must not be used as an argument for postponing preventive action aimed at risk reduction. A risk-benefit analysis must be performed.

- If a number of valid studies of the same species yield different results, the precautionary principle is applied.

\subsection{Positions on the Endocrine Effect of Certain UV Filters}

- Laboratory studies have shown that certain UV filters exert endocrine effects in mammals and fish. UV filters have been demonstrated as residues in ecosystems, fish and the maternal milk.

- UV filters with an endocrine effect are only part of the overall problem of endocrine disruptors.

- The health risk associated with 4-methyl-benzylidene-camphor (4-MBC) is unclear and will be reassessed.

- A close watch must be kept on the riskbenefit ratio in light of current scientific findings: the benefits of protecting skin against sun-induced damage (sunburn, skin cancer, etc.), as compared with the adverse endocrine effects of certain UV filters.

\subsection{Positions on Exposure to and} Protection against Solar Radiation

- Regular, unprotected exposure to solar radiation is considered a health risk that triggers a number of different skin diseases.

- The most important measures for preventing sun-related skin diseases are to seek shade, wear appropriate clothing and use sun screens.
- By skillfully combining different UV filters, the cosmetic industry is able to produce sun screens with high sunprotection factors while using relatively low filter quantities.

\subsection{Recommendations on General Action against Endocrine Disruptors}

- The development of validated test methods for determining endocrine endpoints is to be promoted/supported.

- The endocrine effects of individual chemicals and of mixtures are to be taken into account in assessing the risk to humans and ecosystems.

- If a number of valid studies of the same species yield different results, the precautionary principle is applied.

\subsection{Recommendations on Data- based Action on UV Filters}

- Assessment of the risk to humans requires data

- on the frequency and quantity of sun screen use in the Swiss population, particularly by risk groups (women of childbearing age, children);

- on the concentrations in ecosystems, on the 'internal impact' (monitoring studies, e.g. non-invasive analysis of extraneous substances in human milk) and on the reproductive and developmental toxicity of UV filters in mammals and aquatic organisms.

- These data, as well as data on absorption of UV filters by the skin and the quantities found in the blood (kinetics), have still to be obtained for individual UV filters.

- Data on the location and fate of UV filters in aquatic and terrestrial ecosystems are to be obtained. These data, together with data on bioaccumulation and ecotoxicological effects, provide the basis for assessing ecological risk.

- Data on the accumulation of UV filters in the food chain, particularly on bioaccumulation in fish, are to be obtained.

- Comprehensive ecological risk analyses of UV filters and UV filter mixtures are needed. They require data on the reproductive effects of long-term exposure in fish and other key aquatic and terrestrial organisms.

\subsection{Recommendations on Action on UV Filters by the Authorities}

- The Federal Office for the Environment examines the possibility of banning UV filters on the basis of scientifically substantiated risk assessments and, if required, drafts a corresponding bill. The replaceability of the UV filters proposed for such a ban is taken into account, as are the EU's risk assessments and regulations.
- The Federal Office of Public Health examines the possibility of banning UV filters that are hazardous to health on the basis of a scientifically substantiated risk assessment and, if necessary, adapts the Cosmetics Ordinance, taking account of the EU's risk assessments and regulations.

- The risk to the consumer's health from 4-MBC in sun screens is to be definitively assessed.

- The Federal Office for the Environment and the Federal Office of Public Health examine the revised results of industrial self-monitoring in relation to the ecotoxicology and human toxicology of UV filters.

\subsection{Recommendations on Action} on UV Filters by Industry

- As part of the legally stipulated self-monitoring process, industry must ensure that UV filters do not exert detrimental effects on humans or ecosystems.

- Manufacturers of UV filters revise the self-monitoring process of their UV filters in light of new findings from the NRP 'Endocrine Disruptors' and international research programs (EU) and of findings from relevant peer reviewed scientific publications and submit it to the authorities.

- Manufacturers of UV filters and sun screens exercise their individual responsibility by taking the latest generally accepted scientific findings on the endocrine effects of UV-filters into account in safety assessments of their products.

- Technological opportunities for reducing concentrations of chemical UV filters in sun screens without loss of protective effect are to be further developed or optimally utilized by industry.

- Industry supports the further development of new UV filters, e.g. colloidal UV filters, organic pigments, etc., and of other filters with low toxicological risk potential.

- The formulating industry examines whether it can voluntarily dispense with the use of 4-MBC in sun screens.

\subsection{Recommendations on Action \\ Affording Protection against the Effects of the Sun}

- Consumers are informed on the proper way to behave in sunlight and protect themselves from its effects.

\section{Results of the Consensus Platform 'Brominated Flame Retardants'}

The consensus platform 'Brominated Flame Retardants' involved a total of 19 representatives of the producing and apply- 
ing industries, the authorities and science. They accepted and approved 12 impactrelated positions and 19 action-related recommendations.

\subsection{Positions Regarding the Effect of Flame Retardants}

- Fire is a major source of hazards to life and property. The use of flame retardants, in addition to the selection of suitable materials and designs, constitutes an essential element of fire prevention.

- Fire protection shall be considered in a total view. Benefits as well as impacts shall be considered. Investigation must not be limited to certain specific flameretardant classes or substance groups.

- Flame retardants originating from various chemical classes exist. Brominated flame retardants display beneficial formulation and material properties for certain specific applications such as in plastic housings of electrical and electronic equipment, printed circuit boards and polystyrene foams.

- As a general rule, brominated flame retardants are not the best or most effective flame retardants. Over time, alternative solutions have been developed for many applications.

- Alternative flame retardants must likewise meet stringent requirements governing environmental compatibility and health hazards.

- For various applications, there are currently still no alternatives to brominated flame retardants. This is the case, for example, where laws or customers stipulate particular flame-retardant requirements, such as in the United States. Among other materials, this affects non-polar plastics such as foamed polystyrene.

\subsection{Positions Regarding Brominat- ed Flame Retardants}

- Substance flow analysis is one suitable tool for weighting the effect of substance flows with respect to emissions to the environment. The results of such analysis can serve as the basis for planning actions and measures to reduce environmental emissions.

- Risk assessment of brominated flame retardants must be based on investigation of all components of the technical product (including stereoisomers and byproducts).

- Occupational exposure of workers to brominated flame retardants shall be considered in dedicated studies separate to those targeting effects on consumers. High occupational exposures to brominated flame retardants have been verified among workers in particular in production, disposal and recycling industries.
- TBBPA: Based on our knowledge today, tetrabromobisphenol-A chemically bonded as a copolymer (for example as epoxy resins in printed circuit boards) is considered harmless in the application phase.

\subsection{Position Regarding the Endocrine Activity of Chemicals}

- Both natural as well as synthetic chemicals exist that have endocrine activity.

\subsection{Position Regarding the Endocrine Activity of Brominated Flame Retardants}

- Certain brominated flame retardants have endocrine activity.

- In recent years, the possible impacts of individual brominated flame retardants on human and animal hormone systems have been the subject of increasing investigation. These studies have revealed that various flame retardants have broadly differing impacts on hormone systems.

- PentaBDE is an endocrine disruptor, as antiandrogenous and estrogenous effects were identified in vitro as well as in vivo. Likewise, adverse effects on the thyroid hormone system were observed in several studies.

- Besides foodstuffs, it has been determined that house dust and indoor air are significant sources of PentaBDE ingested and inhaled by humans.

- Based on the data available today, DecaBDE is not an endocrine disruptor, as studies yielded no impacts - neither in vitro nor in vivo - that are directly due to hormone system disruption.

- TBBPA is a potential endocrine disruptor. Multiple in vitro studies yielded impacts on the thyroid hormone system. However, these findings were not confirmed for TBBPA in vivo.

- HBCDs are potential endocrine disruptors, as several in vitro studies produced low-level interactions with the thyroid hormone system. These findings are currently the subject of experimental testing in vivo.

- The knowledge gained from cell and animal testing within the scope of these studies cannot be directly applied to human beings. The relevance of experimental findings for human health has therefore not yet been comprehensively clarified.

- As in vitro and in vivo investigations have demonstrated, certain brominated flame retardants (PentaBDE, HBCD and DecaBDE) are capable of disrupting neuron development in experiments conducted with test animals. To what extent this knowledge is transferable to humans is currently the subject of scientific study.
- Risk assessments of major brominated flame retardants (TBBPA und HBCD) are currently underway in the EU; conclusive reports on individual substances (DecaBDE, OctaBDE and PentaBDE) have already been completed. An addendum on DecaBDE is currently being prepared to the report on the environmental risk assessment from the year 2004. Knowledge gaps still exist with respect to the possible impacts that the remaining brominated flame retardants (besides PentaBDE, DecaBDE, TBBPA and HBCDs) have on endocrine systems.

\subsection{Recommendations on General Actions to be Taken with Regard to Endocrine Disruptors}

- The problems created by endocrine active substances necessitate long-term monitoring and research projects.

- Mindful of the magnitude of the potential hazard posed by endocrine-active substances, we believe that scientific uncertainty must not be allowed to serve as an argument for putting off appropriate action to reduce risk

\subsection{Recommendations on General Actions to be Taken with Regard to Brominated Flame Retardants}

- Efforts in search of alternatives to brominated flame retardants that persist in the environment must be promoted.

- Endocrine disruptors are covered in among other legislation, the European chemical regulation REACH (Registration, Evaluation, Authorization and Restriction of Chemicals). Brominated flame retardants are used worldwide. It is therefore inappropriate to make do with a focal point that considers Switzerland alone.

- We must improve our knowledge of the lifecycle of brominated flame retardants.

- Investigations must focus on the substance flows and lifecycles of brominated flame retardants. This also includes production data, substance quantities in products, recycling and in particular the emissions occurring during use.

- Analysis of the occupational exposures of recycling industry workers to brominated flame retardants shall serve to clarify where exposures are highest and whether action to reduce exposure is needed.

- DecaBDE: Emissions to the environment of DecaBDE within the course of product technical lifecycle must be investigated.

- DecaBDE: Detailed information on the production of DecaBDE and its use in various materials as well as its con- 
sumption must be made known (substance flow analysis).

- DecaBDE: In order to better understand the enrichment of DecaBDE and other brominated flame retardants along food chains, birds of prey, their eggs and their prey must be studied and tested for the presence of these substances and their related degradation products.

- HBCD: Giving due consideration to ongoing scientific investigations, it must be clarified whether alpha-HBCD is formed in the environment from technical HBCD or is selectively transported and/or ingested, inhaled or absorbed by living organisms.

- TBBPA, HBCD and DecaBDE: To enable better assessment of the exposures incurred by living organisms in aquatic and terrestrial ecosystems, the degradation behaviour of substances and their degradation products in sewage sludge, sediments and soil should be more thoroughly investigated taking into account scientific studies presently already underway.

- alpha-HBCD: Owing to the significant enrichment of alpha-HBCD in the biota (in the biological environment) and the hazards this substance is known to pose today, appropriate measures must be considered with the goal of reducing emissions.

\subsection{Recommendations on Regula- tory Authority Actions to be Taken with Regard to Brominated Flame Retardants}

- Based on scientifically based risk assessments, regulatory authorities are reviewing whether to impose further restrictions or bans on persistent and hormone-active brominated flame retardants. Such review also considers pertinent EU regulations and the availability of substitute substances subject to equally stringent investigation.

- DecaBDE and TBBPA: A risk assessment report on DecaBDE and TBBPA has been completed. Based on the investigations called for in this report and on the outcome of current, ongoing research work, the regulatory authorities aim to introduce, where needed, regulation of these substances and/or measures to reduce risk. This is being done in coordination and agreement with the corresponding EU regulations.

\subsection{Recommendations on Industry Actions to be Taken with Regard to Brominated Flame Retardants}

- The processing and disposal industries are investigating whether relevant emission sources of brominated flame retardants exist in Switzerland, and initiating emissions mitigation measures where appropriate.

- DecaBDE: Emissions to the environment of DecaBDE within the course of the technical lifecycle of products must be reduced. Industry has introduced a voluntary initiative, VECAP, for the very purpose of reducing emissions.

- DecaBDE: Efforts being made for the Product Stewardship Program with respect to DecaBDE should be fostered and supported. This applies in particular to cooperation between producers and user industries, as well.

- DecaBDE and HBCD: Industry is dedicating efforts within the scope of exercising self-responsibility to identify areas of application in which DecaBDE and HBCD can be replaced with other flame-retardant systems possessing more favourable risk profiles.

\section{Results of the Consensus Platform 'Endocrine Disruptors in Wastewater and in the Aquatic Environment'}

The consensus platform 'Endocrine Disruptors in Wastewater and in the Aquatic Environment' involved a total of 15 representatives of the industry, the authorities and science. They accepted and approved 32 impact-related positions and 20 actionrelated recommendations.

\subsection{Positions Regarding (Potential) Endocrine Disruptors in the Aquatic Environment}

- Chemicals and chemical groups (primary substances and metabolites) that can have an endocrine effect have been detected in Swiss rivers and streams.

- Endocrine disruptors are carried into aquatic systems both from point sources (e.g. wastewater treatment plants) and from diffuse sources (e.g. surface rainwash or dry deposition).

- Even compounds that break down easily, such as the steroid hormone estradiol, can be permanently present in low concentrations in water bodies downstream of waste water treatment plants ('pseudo-persistence').

- Field studies conducted all over the world show that the occurrence of endocrine disruptors in the aquatic environment leads to local impacts as well.

- The impact of pollution peaks (e.g. from pulse pollution events as a result of sewer system discharges) is not clear.

- In Switzerland, endocrine disruptors in the aquatic environment represent a problem at hot spots and at regional level, especially where dilution of the treated waste water in the receiving water is not sufficient (1:10 or more), either at certain times of year or all year round. This can be the case in densely populated regions and with waste water treatment plants that feed into small streams.

- Research in Switzerland has shown that fungal metabolites with endocrine activity (e.g. the highly potent zearalenone) can enter surface waters from cereal crops, but are usually quickly diluted to below the effect concentration.

\subsection{Positions Regarding (Potential) Endocrine Disruptors in Waste- water}

- Wastewater treatment plants play a key role in the emission of steroid hormones and lead to their constant release into the aquatic environment. The construction standard of wastewater treatment plants has a decisive influence on emissions.

- It is not really known what part discharges of untreated wastewater from sewage systems (pulse pollution events) play in endocrine activity in the aquatic environment and what the resultant effects are on aquatic life.

- In treated domestic wastewater, it is natural and synthetic steroid hormones that are responsible for the majority of the estrogen activity. Overall, estrogen contamination of the aquatic environment is primarily from this source.

- Wastewater discharges must not result in effects in rivers or streams due to estrogen substances from the wastewater (protection objective). According to our current knowledge, the appropriate bioindicator for this is the identification of increased vitellogenin levels in male fish.

\subsection{Positions Regarding (Potential) Endocrine Disruptors in Drinking Water}

- The steroid hormone concentrations in drinking water measured in scientific studies in Germany are not critical from a health point of view. Contamination via drinking water is several orders of magnitude smaller than the body's own hormone production.

- It can be assumed that in general the endocrine disruptors ingested via drinking water are not critical for human health in Switzerland either. Isolated cases of higher contamination of groundwater and drinking water must be assessed on a case-by-case basis.

- Certain substances (bisphenol A, phthalates) that mimic estrogen can come not only from environmental pollution of groundwater and drinking water, but also from materials that are in contact with drinking water. Their activity is, however, much lower than that of estradiol. 
7.4. Positions on the Impact of Endocrine Disruptors on Aquatic Life

- There are chemicals that trigger adverse effects by disrupting the human and animal hormone system.

- Different organisms exhibit different sensibilities to endocrine disruptors.

- Even invertebrates are sensitive to endocrine disruptors (e.g. imposex in water snails).

- The consequences of the impact of endocrine disruptors for ecosystems and biodiversity are not yet foreseeable.

- Similarly, the consequences of combinations of stressors, such as parasite stress, poor habitat and physical parameters, on the hormonal impact are largely unknown. There is a clear need for research at this level.

- As shown by in vitro tests, the estrogen potency of natural and synthetic steroid hormones is several orders of magnitude greater than that of previously investigated industrial chemicals.

- In the case of some chemicals (e.g. nonylphenolpolyethoxylates) it is only the degradation products that exhibit endocrine activity.

- Experimental studies show that mixtures of various endocrine disruptors have an endocrine impact, even where no impact can be demonstrated for the individual components in the concentrations present (cumulative effect).

- Laboratory experiments on aquatic invertebrates, fish and amphibians show clearly that environmental substances disrupt the hormone balance of such organisms. Chronic exposure to endocrine disruptors at environmentally relevant concentrations can lead to adverse changes in development, growth and reproduction.

- All over the world, hormone-like impacts have been observed in fish that are exposed to treated wastewater from mechanical-biological wastewater treatment plants.

- Organisms (fish) show effects especially when continually exposed to even very low concentrations of endocrine disruptors (e.g. increased vitellogenin levels).

- Effects on organisms (both the type of effect and effect thresholds) are dependent on the lifecycle of the organism. For instance, the gonadal differentiation phase in fish is very sensitive to exposure to endocrine disruptors.

- In a minority of the Swiss water bodies studied to date, slight to moderate increases in vitellogenin values were measured in male fish in the impact zone of wastewater treatment plants, which suggests exposure to estrogen. Apart from this, there are few indications of any impact of endocrine disruptors in Swiss surface waters.
- So far, apart from a very few exceptions, only incomplete impact data are available in Switzerland indicating that exposure to endocrine disruptors under environmental conditions leads to undesirable biological changes.

- So far there are no indications that the high prevalence of gonadal abnormality in whitefish in Lake Thun is attributable to endocrine disruptors.

\subsection{Positions on Test Systems and Quality}

- Vertebrates all react in a similar way to steroid hormones and xenoestrogens. It appears unlikely, though, that all invertebrates also react in the same way to estrogens. It is therefore impossible in practice with current test procedures to establish quality criteria that protect all organisms in the environment against adverse effects.

- Acute toxicity tests are usually not suitable for predicting chronic effects.

- The total contamination of water bodies with endocrine disruptors could be measured using biotests in specialized laboratories. Estrogens and androgenic contamination are already measured in this way. For total assessments they are therefore to be preferred to an analysis of individual substances.

\subsection{Recommendations on General Actions}

- The risks posed by endocrine disruptors call for long-term monitoring and research projects.

- Scientific research in the area of endocrine disruptors should be promoted generally. Further fundamental research is necessary, particularly into the potential effects of endocrine disruptors on the human and animal organism so that a comprehensive risk assessment can be conducted.

- There is a clear need for research into the following questions:

- How do endocrine disruptors affect biological functions outside of the reproductive system?

- Can experimental results relating to specific modes of action be transferred from one species to the others?

- What heritable impacts on gene regulation and gene expression do endocrine disruptors have (epigenetic consequences)?

- What impact do endocrine disruptors have on sensitive development stages? Could this impact be behind later diseases such as cancer or immune deficiency?

- What impact does the combination of stressors have on aquatic life?

- The environmental behavior of micropollutants needs to be investigat- ed in greater detail. In Switzerland, chemical flows of endocrine disruptors need to be systematically analyzed and modeled in order to identify relevant exposure situations. It will then be possible to derive impact-related actions based on scientifically grounded risk assessments.

- Fish and other aquatic organisms should be studied to establish the biological impact of the environmental stress calculated by means of chemical analyses or forecast using models.

- When carrying out risk assessments for technical mixtures, the interaction of the components and degradation products should be studied where possible, as well as their individual environmental behavior. Their complex composition and the different toxicity levels of the key individual components should also be considered.

\subsection{Recommendations on Actions Concerning (Potential) Endocrine Disruptors in Wastewater and in the Aquatic Environment}

- It is highly probable that estrogenic steroid hormones are the primary contributors to this kind of hormone activity in the aquatic environment. This means that they are to be viewed as being primarily responsible for any estrogen effects in fish. In practice, therefore, the introduction of a quality objective for estrogen activity in the aquatic environment is very important. This applies particularly to sensitive rivers and streams affected by wastewater treatment plants in which the treated wastewater is insufficiently diluted.

- Whenever possible, treated wastewater from wastewater treatment plants should be fed into a receiving body of water that ensures a sufficiently high dilution ratio.

- The construction of wastewater treatment plants should permit optimum elimination of the substances contained in the wastewater. It must be ensured that the plants function as well as possible. Problems can arise as a result of micropollutants, particularly where the treated wastewater in the water body is insufficiently diluted. In such cases, the following measures should be examined:

- State-of-the-art construction

- Further technical measures (activated carbon, ozonation, increasing sludge age, etc.)

- Alternative courses of action, e.g. creation of integrated waste water networks so that more efficient wastewater treatment plants can be established 


\subsection{Recommendations on Regulatory Authority Actions Concerning (Potential) Endocrine Substances in Wastewater and in the Aquatic Environment}

- The regulation and implementation of actions in urban drainage should be based on integrated strategy and planning. The key causes of the pollution of the aquatic environment at catchment area level should be taken into account and prioritized, and actions should be identified that represent optimum benefit for the aquatic environment at an economically affordable cost (including agricultural actions, renaturation, etc.). Measures for optimizing rainwater and sewer system management (discharges, leakage losses) should also be assessed. As well as reducing endocrine disruptors, the intended actions for wastewater treatment plants also reduce contamination by other substances relevant for the aquatic environment.

- Wastewater treatment plants in Switzerland are to be reviewed in terms of their elimination performance and their technical optimization potential.

- Actions at source, such as alternative systems for urban drainage, hospital wastewater treatment or the NoMix toilet from EAWAG, need to be evaluated and, if appropriate, promoted.

\subsection{Recommendations on Industrial Actions Concerning (Potential) En- docrine Disruptors in Wastewater and in the Aquatic Environment \\ - If a risk is identified for a particular} substance or particular groups of substances in an officially recognized risk assessment, industry takes the relevant precautions to reduce or eliminate this risk.

- Industry endeavors to replace substances that have a significant toxicological or ecotoxicological risk with lower-risk substances. For this to work, the substitute products need to be subjected to a risk assessment as thorough as that applied to the substances they are designed to replace.
7.10. Recommendations on Agricultural Actions Concerning (Potential) Endocrine Disruptors in Wastewater and in the Aquatic Environment

- Site-appropriate management of agricultural areas limits contamination by endocrine disruptors and other substances via surface rain-wash and drainage.

- The selection of suitable cereal varieties and optimized working of harvest residues to promote rotting can minimize environmental contamination by the fungal endocrine disruptor zearalenone and its metabolites.

\subsection{Recommendations on Actions} for Developing Test Systems and

\section{Quality Criteria}

- Test methods are to be established for prioritizing micropollutants and for monitoring/assessing the quality of water bodies.

- Strategies for recording pollution of the aquatic environment can be based on models and/or biological screening. Pollution hotspots are to be verified using chemical analysis investigations of water bodies.

- The rapid development of internationally (OECD) recognized methods for detecting endocrine-disruptor characteristics of chemicals and mixtures must be promoted.

- For practical application, efforts should be made to establish scientifically grounded quality criteria for hormone activity in the aquatic environment: a chronic quality criterion (CQC) for long-term contamination and an acute quality criterion (AQC) for short-term contamination peaks. It is recommended that these quality criteria be added to the Swiss water protection ordinance (Gewässerschutzverordnung).

\section{Conclusions}

The methodology of the consensus platform has proven successfully its practicability in converting scientific findings into generally accepted recommendations as well as political and practical action. With their joint effort to prepare impactand action-related statements, representatives from producing and applying industry, regulatory authorities and scientists of the National Research Program 'Endocrine Disruptors' participated in a constructive dialogue, thereby making a very valuable contribution towards preventing negative impacts of endocrine disruptors. Thus, the consensus platforms are the key implementation element of this National Research Program. The same methodology can be applied - maybe in an adapted way - whenever various stakeholder groups willing to cooperate are looking for a best general agreement.

\section{Acknowledgements}

The author expresses his gratitude to the SNSF to grant the development and organization of the consensus platforms (grant nr. 4050-068561) and thanks the members of the consensus platforms for their highly valued participation (in alphabetical order): Gabriele Allard, Felix R. Althaus, Judith Amberg-Müller, Claude Bastian, Jürgen Baumann, Jochen Bausch, Adrian Beard, Lasse Braathen, Beat Brüschweiler, Thomas Bucheli, Andreas Buser, Peter Dollenmeier, Michel Donat, Helmut Elbert, Karl Fent, Urs Friederich, Hans-Jürg Furrer, René Gälli, Andreas Gerecke, Walter Giger, Patricia Holm, Bernhard Irrgang, Georg Karlaganis, Hans-Peter Kohler, Martin Kohler, Marcel Langenauer, Walter Lichtensteiger, Leo Morf, Stephan Müller, Ueli Ochsenbein, Uli Osterwalder, Ferdinand Quella, Klaus Rothenbacher, Christoph Rüegg, Michael Schärer, Margret Schlumpf, Peter Schmid, Helmut Segner, Christoph Studer, Marc J.-F. Suter, Josef Tremp, Paul Vesel, Michael Weller, Steffen Wengert, Martin Würsten.

Received: March 22, 2008 